\title{
A study of Neurological Soft Signs and Minor Physical Anomalies in children of parents with Schizophrenia and those without Schizophrenia
}

\author{
Gaurav Wadgaonkar ${ }^{1}$, Mansi Jain ${ }^{2}$, Pratik Surandashe ${ }^{3}$, Avinash De Sousa ${ }^{4}$ \\ ${ }^{1}$ Senior Resident Doctor, Smt. Kashibai, Navale Medical College, Pune. \\ ${ }^{2}$ Specialty Medical Officer, Department of Psychiatry, Lokmanya Tilak Municipal Medical College, \\ Mumbai. \\ ${ }^{3}$ Specialty Medical Officer, Department of Psychiatry, Topiwala National Medical College and BYL Nair \\ Charitable Hospital, Mumbai. \\ ${ }^{4}$ Research Associate, Department of Psychiatry, Lokmanya Tilak Municipal Medical College, Mumbai.
}

Corresponding author: Mansi Jain

Email-drjainmansi88@gmail.com

\begin{abstract}
Introduction: 'Neurological soft signs (NSS)' and 'Minor physical anomalies (MPA)' represent two quite distinct markers of risk for schizophrenia that stem from genetic factors and have been studied over the past 3 decades. The aim of the current study was to assess Neurological soft signs (NSS) and Minor physical anomalies (MPA) in children of patients suffering from schizophrenia and compare the same to children having parents without any psychiatric disorders.

Methodology: The study was a cross sectional study at a Private tertiary care psychiatric hospital in urban set up. The data was collected over a period of 1 year during June 2016- June 2017. The subjects of this study were children of parents suffering from schizophrenia and children of parents without any psychiatric disorder. The control group was made up of 40 children of the same age group with parents having no psychiatric disorders. Following the collection of sociodemographic details using a semistructured proforma, children in both groups were assessed using the Physical and Neurological Soft sign Scale (PANESS) while MPAs were assessed using the Waldrop scale. The data collected was statistically analyzed.

Results: There was no statistically significant correlation between demographic factors like gender, age, birth order, educational status or type of delivery with neurological soft signs and minor physical anomalies. It was found that the study group had higher score for incoordination of gait and balance; higher score for slowness in performing patterned speed of movements which included toe heel alteration, hand supination and pronation, finger succession as compared to control group along with higher scores for involuntary movements, excess dysrhythmia score and overflow score including mirror movements in index group than controls. There was no statistically significant difference in lateral preference patterns or mix handedness of cases and controls. Among Minor physical anomalies, the study group also had more anomalies in mouth like high-steeped palate and tongue anomaly, curved $5^{\text {th }}$ finger of hands and big gap between first and second toe (sandal gap).

Conclusion: NSS and MPA are important variables and biomarkers that may be assessed in patients with schizophrenia and their offspirngs.
\end{abstract}

Key words: neurological soft signs, minor physical anomalies, schizophrenia, children.

(Paper received $-10^{\text {th }}$ March 2018, Peer review completed $-26^{\text {th }}$ March 2018)

(Accepted $-29^{\text {th }}$ March 2018) 


\section{INTRODUCTION}

Debate still prevails on the etiology of schizophrenia as a developmental disorder due to genetic factors or a neurodegenerative disorder due to environmental factors present in an individual's life [1]. Data from family, twin and adoption studies show that even though first degree relatives of patients may not develop the disease itself, there is familial aggregation of genetic factors responsible for schizophrenia [2-3]. 'Neurological soft signs (NSS)' and 'Minor physical anomalies (MPA)' represent two quite distinct markers of risk for schizophrenia that stem from genetic factors and have been studied over the past 3 decades [4].

NSS refers to any neurological deviation, motor, sensory or integrative that does not localize the site of putative CNS lesion and thought to result from intrauterine or perinatal injury or anoxia [5]. Simply put, these are subtle but observable neurological impairments in motor and sensory functions which cannot be localized to any specific area of the brain and do not characterize any specific neurological disorder [6]. Some of the common NSS include agraphaesthesia, stimulus extinction, right-left confusion, hopping, finger-thumb opposition, dysdiachokinesis, finger-to-nose test etc [7]. Increased frequency of NSS is associated with a myriad of psychiatric disorders ranging from schizophrenia, obsessive compulsive disorder, post traumatic stress disorder, attention deficit hyperactivity disorder, bipolar disorder and learning disability to other affective disorders [8-11]. First degree relatives of patients suffering from schizophrenia have shown an increased prevalence of NSS compared to controls [12]. The prevalence of NSS amongst patients having schizophrenia in the Indian population was found to be around $65 \%$, while a recent meta-analytic study found the prevalence to be around 50-73\% [13-14].

MPAs are deviations in external physical characteristics that are ectodermal in origin. These include slight dysmorphic features representing subtle alterations in the development of various bodily structures in the mouth, eye, ear, global head, hand, and feet areas. Since the central nervous system (CNS) also derives from ectodermal tissue, the presence of excess physical anomalies may be related to abnormal development of the CNS [15]. There is an increased prevalence of MPAs in patients with schizophrenia, their non-affected siblings and first degree relatives when compared to normal populations especially in the eye, mouth and hand/foot regions [16-17]. The prevalence of MPA in patients with schizophrenia is reported to be as high as $40-60 \%$ [16].

Both NSS and MPA are considered separate etiological endophenotypes in schizophrenia [17]. Since endophenotypes are present before the onset of disease and in individuals with heritable risk for disease such as unaffected family members, they can be used to help diagnose and search for causative genes and factors in the genesis of the illness. The aim of the current study was to assess Neurological soft signs (NSS) and Minor physical anomalies (MPA) in children of patients suffering from schizophrenia and compare the same to children having parents without any psychiatric disorders.

\section{METHODOLOGY}

Study setting and background: The study was a cross sectional study at a Private tertiary care psychiatric hospital in urban set up. The data was collected over a period of 1 year during June 2016- June 2017.

Subjects: The subjects of this study were children of parents suffering from schizophrenia and children of parents without any psychiatric disorder. 40 consecutive children between the age group of 5-15 years having at least one parent diagnosed with schizophrenia without any other major psychiatric co-morbidity (except nicotine dependence) attending the out-patient clinic were enrolled for the study. The control group was made up of 40 children of the same age group with parents having no psychiatric disorders. Children themselves under evaluation for or diagnosed with major medical, psychiatric and neurological disorders were excluded from the study. The parents were explained the aims and objectives of the study and a written informed assent from children and consent from parents was taken. 


\section{Tools used in the assessment}

Following the collection of sociodemographic details using a semi-structured proforma, children in both groups were assessed using the Physical and Neurological Soft sign Scale (PANESS) while MPAs were assessed using the Waldrop scale. Assessment of each child for all the above was done in a single sitting.

1. Physical and Neurological Soft sign Scale (PANESS): It is scale for assessing physical and neurological soft signs in children and adolescents. It is an observational scale having 21 questions covering gait, stance, laterality, quality of rapid movements, impersistence score, involuntary movement score, repetitive speed of movement score, and sequenced speed of movement score, asymmetrical movement score. It assesses in terms of laterality, timed and untimed motor movements.It is found to have adequate test retest reliability, inter-rater reliability and internal consistency [18].

2. Waldrop scale: It is a standardized scoring system for assessing the incidence of minor physical anomalies. The Original Waldrop scaleused in the study includes various parameters for anomalies of head, eyes, ear, hand and feet, in total 18 MPAs are studied. The minimum score is 0 while the maximum score is 24 [19].

\section{STATISTICAL ANALYSIS}

Quantitative data has been presented with the help of Mean, Standard deviation, Median and Interquartile range (IQR). Comparison pre and post has been done with the help of Unpaired T test or Mann-Whitney test as per results of normality test. Qualitative data is presented with the help of Frequency and Percentage table. Association among study group is assessed with the help of Chi Square test. P value less than 0.05 was taken as significant level. The entire statistical analysis has been done using Graph Pad Computerized Software.

\section{RESULTS}

\section{Sociodemographic Factors}

Studying the socio-demographic factors, 26 male and 16 female children were found in the case group, while 25 male and 15 female children were in the control group. Both the groups did not differ significantly in educational levels or other socio-demographic parameters. There was no statistically significant correlation between demographic factors like gender, age, birth order, educational status or type of delivery with neurological soft signs and minor physical anomalies.

\section{Data on NSSs and MPAs}

Our study found higher frequency of Neurological soft signs (NSS) and Minor Physical Anomalies (MPAs)in children of parents suffering from schizophrenia than children of normal parents.Among the neurological soft signs (Table 1), we found our index group had higher score for incoordination of gait and balance; higher score for slowness in performing patterned speed of movements which included toe heel alteration, hand supination and pronation, finger succession as compared to control group along with higher scores for involuntary movements, excess dysrhythmia score and overflow score including mirror movements in index group than controls. There was no statistically significant difference in lateral preference patterns or mix handedness of cases and controls. Among Minor physical anomalies (Table 2), index group also had more anomalies in mouth like high-steepled palate and tongue anomaly, curved $5^{\text {th }}$ finger of hands and big gap between first and second toe (sandal gap).

\section{DISCUSSION}

Varied pattern of lateral preference and mixed handedness have been reported by researchers across different populations, the absence of statistically significant finding in our study may suggest the need to assess the same in a larger population to find any differences among western and Indian population [1920]. Presence of neurological soft signs and minor physical anomalies in a greater frequency among the 
children of patients suffering from schizophrenia supports the neurodevelopmental hypothesis of schizophrenia. Higher frequency of NSS and other neurological abnormalities has been reported in offspring of mothers suffering from other psychotic disorders as well [20].

Higher NSS and MPAs in healthy first degree relatives of patients with schizophrenia than normal population were reported by researchers and they found Minor physical anomalies of hand region like curved $5^{\text {th }}$ finger and transverse palmar crease more in first-degree relatives of patients with schizophrenia $[4,6]$. Higher incidence of high-palate and big gap between first and second toe was reported by other authors [21]. Researchers have also found a more high-steeped palate and epicanthus anomalies in siblings of schizophrenia patients [15]. However, presence of MPA in eyes and ears region found by previous studies was not replicated in our study, emphasizing the need to study differences in MPAs across different cultures. Presence of similar findings in different cultures goes to show that etiology of schizophrenia remains same across all cultures. Neurological soft signs are associated with regional grey matter volume changes like reduced gray matter densities in the precentral and postcentral gyri, the inferior parietal lobule and the inferior occipital gyrus; and may represent a clinical sign of the perturbed cortical-subcortical connectivity that is hypothesized to underlie psychotic disorders [22-23]. Similarly MPAs are proposed to occur as a result of intrauterine insult during 'organogenesis' i.e. first trimester of pregnancy [24]. These neurodevelopmental insults at around first or early second trimester may lead to the activation of pathologic neural circuits during adolescence or young adulthood which causes positive or negative symptoms of schizophrenia [25].

Table 1: Comparison of NSS in children of parents having schizophrenia and children of parents without schizophrenia

\begin{tabular}{|c|c|c|c|}
\hline & Cases(40) & Controls(40) & $\mathrm{p}$ value \\
\hline \multicolumn{4}{|l|}{ Lateral preference \# } \\
\hline Right & 37 & 33 & \multirow[t]{3}{*}{0.256} \\
\hline Left & 3 & 5 & \\
\hline Mixed & 0 & 2 & \\
\hline Mean GBTE score* & $1.78 \pm 2.236$ & $0.78 \pm 2.567$ & $0.0001^{*}$ \\
\hline \multicolumn{4}{|l|}{ Mean QMDE score* } \\
\hline Total & $5.85 \pm 5.28$ & $2.40 \pm 2.56$ & $0.02^{*}$ \\
\hline Left & $3.08 \pm 2.89$ & $1.08 \pm 1.34$ & $0.004^{*}$ \\
\hline Right & $2.78 \pm 2.46$ & $1.33 \pm 1.28$ & $0.031^{*}$ \\
\hline Mean IS score* & $0.10 \pm 0.30$ & $0.05 \pm 0.22$ & 0.399 \\
\hline Mean IM score* & $0.25 \pm 0.54$ & $0.03 \pm 0.15$ & $0.013^{*}$ \\
\hline \multicolumn{4}{|l|}{ Mean RSM score* } \\
\hline Total & $4.88 \pm 4.63$ & $3.53 \pm 3.37$ & 0.41 \\
\hline Left & $2.49 \pm 2.38$ & $1.69 \pm 1.64$ & 0.265 \\
\hline Right & $2.45 \pm 2.36$ & $1.88 \pm 1.82$ & 0.519 \\
\hline \multicolumn{4}{|l|}{ Mean PSM score* } \\
\hline Total & $6.73 \pm 5.39$ & $4.23 \pm 3.18$ & $0.02^{*}$ \\
\hline Left & $3.30 \pm 2.69$ & $2.08 \pm 1.57$ & $0.041^{*}$ \\
\hline Right & $3.43 \pm 2.71$ & $2.15 \pm 1.64$ & $0.024^{*}$ \\
\hline \multicolumn{4}{|l|}{ Mean OG score* } \\
\hline Total & $1.53 \pm 1.64$ & $0.48 \pm 1.01$ & $0.001^{*}$ \\
\hline Left & $0.85 \pm 1.00$ & $0.35 \pm 0.77$ & $0.007^{*}$ \\
\hline Right & $0.68 \pm 1.07$ & $0.13 \pm 0.33$ & $0.006^{*}$ \\
\hline Mean ORM score* & $0.93 \pm 1.32$ & $0.28 \pm 0.81$ & $0.001^{*}$ \\
\hline Mean OPM score* & $3.20 \pm 2.78$ & $1.90 \pm 1.97$ & 0.066 \\
\hline
\end{tabular}

[In the variable column, \#- Pearson chi square test, *Mann -Whitney test]

[GBTE= gait and balance total error, QMDE=quality of movement dysrhythmia error, IS= impersistence score, IM= involuntary movement score, RSM= repetitive speed of movement, PSM= patterned speed of movement, $\mathrm{OG}=$ overflow in gait, $\mathrm{ORM}=$ overflow in repetitive movements, $\mathrm{OPM}=$ overflow in patterned movement.]

(In the $\mathrm{p}$ value column, * significant $-\mathrm{p}<0.05$ ) 
Table 2: Comparison of MPAs in children of parents having schizophrenia and children of parents without schizophrenia

\begin{tabular}{|c|c|c|c|}
\hline MPA & $\begin{array}{c}\text { Cases } \\
\text { (mean score) }\end{array}$ & $\begin{array}{c}\text { Control } \\
\text { (mean score) }\end{array}$ & $p$ value \\
\hline \multicolumn{4}{|l|}{ Eyes* } \\
\hline Epicanthus & $0.23 \pm 0.42$ & $0.13 \pm 0.33$ & 0.24 \\
\hline Hypertelorism & $0.15 \pm 0.36$ & $0.13 \pm 0.33$ & 0.74 \\
\hline \multicolumn{4}{|l|}{ Ears* } \\
\hline Low seated ears & $0.05 \pm 0.22$ & $0.00 \pm 0.00$ & 0.15 \\
\hline Adherent ear lobes & $0.03 \pm 0.15$ & $0.10 \pm 0.30$ & 0.16 \\
\hline \multicolumn{4}{|l|}{ Mouth * } \\
\hline High steepled palate & $0.15 \pm 0.48$ & $0.00 \pm 0.00$ & $0.04^{*}$ \\
\hline Tongue anomaly & $0.10 \pm 0.30$ & $0.00 \pm 0.00$ & $0.04^{*}$ \\
\hline \multicolumn{4}{|l|}{ Hands* } \\
\hline Curved $5^{\text {th }}$ finger & $0.23 \pm 0.53$ & $0.03 \pm 0.15$ & $0.02^{*}$ \\
\hline Single palmar crease & $0.00 \pm 0.00$ & $0.00 \pm 0.00$ & \\
\hline \multicolumn{4}{|l|}{ Feet* } \\
\hline $3^{\text {rd }}$ toe abnormality & $0.03 \pm 0.15$ & $0.03 \pm 0.15$ & 1 \\
\hline Big gap between $1^{\text {st }} \& 2^{\text {nd }}$ toe & $0.48 \pm 0.50$ & $0.23 \pm 0.42$ & $0.02^{*}$ \\
\hline Total MPA score $\$$ & $1.43 \pm 1.83$ & $0.63 \pm 0.89$ & $0.019 *$ \\
\hline
\end{tabular}

[In the variable column, *- Mann Whitney test, \$ Unpaired T test]

( $\mathrm{p}$ value column, ${ }^{*} \mathrm{p}<0.05=$ significant $)$

NSS in first-degree relatives of schizophrenia patients may suggest 'endophenotype' criterion of familial association namely: association with the illness and state independence (occurs even without clinical episodes of disease).Use of endophenotypes in psychiatry can lead to successful genetic analysis of psychiatric disorders [26-27]. Finally NSS and MPA can serve as vulnerability markers for at risk individuals. This study attempts to provide data from Indian perspective for future research on finding independent risk markers or endophenotypes in schizophrenia.

\section{REFERENCES}

1. Szulc A, Galińska B, Czernikiewicz A. Schizophrenia--neurodevelopmental and neurodegenerative theory revisited. Psychiatria Polska 2005;39(2):249-58.

2. Kendler KS, McGuire M, Gruenberg AM, Walsh D. An epidemiological, clinical and family study of simple schizophrenia in County Roscommon, Ireland. Am J Psychiatry 1994;151:27-34.

3. Tsuang MT, Gilbertson MW, Faraone SV. The genetics of schizophrenia. Current knowledge and future directions. Schizophr Res 1991;4:157-71.

4. Compton M, Bollini AM, Mack LM, Kryda AD, Rutland J, Weiss PS et al. Neurological soft signs and minor physical anomalies in patients with schizophrenia and related disorders, their first-degree biological relatives, and non-psychiatric controls. Schizophr Res 2007;94:64-73

5. Chan RCK, Gottesman I. Neurological soft signs as candidate endophenotypes for schizophrenia: A shooting star or a Northern star? Neurosci Biobehav Rev 2008;32(5):957-71.

6. Bombin I, Arango C, Buchanan RW. Significance and meaning of neurological signs in schizophrenia: two decades later. Schizophr Bull 2005;31(4):962-77.

7. Varambally S, Venkatasubramanian G, Gangadhar BN. Neurological soft signs in schizophrenia - The past, the present and the future. Indian J Psychiatry 2012;54(1):73-80.

8. Cox CS. Neuropsychological abnormalities in obsessive-compulsive disorder and their assessment.1997;9(1):45-60.

9. Gurvits TV, Gilbertson MW, Lasko NB, Tarhan AS, Simeon D, Macklin ML et al. Neurologic Soft Signs in Chronic Posttraumatic Stress Disorder. Arch Gen Psychiatry 2000;57(2):181-6.

10. Patankar VC, Sangle JP, Shah HR, Dave M, Kamath RM. Neurological soft signs in children with attention deficit hyperactivity disorder. Indian J Psychiatry 2012;54(2):159-65.

11. Punt M, DE Jong M, DE Groot E, Hadders-Algra M. Minor neurological dysfunction in children with dyslexia. Dev Med Child Neurol 2010;52(12):1127-32.

12. Solanki RK, Swami MK, Singh P, Gupta S. Identification of vulnerability among first-degree relatives of patients with schizophrenia. East Asian Arch Psychiatry 2012;22(3):118-25.

13. Venkatasubramanian G. Schizophrenia is a disorder of aberrant neurodevelopment: A synthesis of evidence from clinical and structural, functional and neurochemical brain imaging studies. Indian J Psychiatry 2007;49(4):244-9. 
14. Chan RCK, Ting Xu, Heinrichs RW, Yue Yu, Ya Wang. Neurological Soft Signs in Schizophrenia: A Metaanalysis. Schizophr Bull 2010;36(6):1089-104.

15. Ismail B, Cantor-Graae E, McNeil TF. Minor physical anomalies in schizophrenic patients and their siblings. Am J Psychiatry 1998;155(12):1695-702.

16. Gourion D, Goldberger C, Bourdel MC, Jean Bayle F, Lôo H, Krebs MO. Minor physical anomalies in patients with schizophrenia and their parents: prevalence and pattern of craniofacial abnormalities. Psychiatr Res 2004; 125(1):21-8

17. Schubert EW, McNeil TF. Prospective Study of Neurological Abnormalities in Offspring of Women with Psychosis: Birth to Adulthood. Am J Psychiatry 2004;161(6):1030-7.

18. Guy W. Physical and neurological examination for soft signs (PANESS). ECDEU Assessment Manual for Psychopharmacology. Revised ; 1976.

19. Sivkov ST, Akabaliev VH. Minor physical anomalies in mentally healthy subjects: internal consistency of the Waldrop Physical Anomaly Scale. Am J Hum Biol 2003;15(1):61-7.

20. Egan MF, Hyde TM, Bonomo JB, Mattay VS, Bigelow LB, Goldberg TE. Relative Risk of Neurological Signs in Siblings of Patients With Schizophrenia. Am J Psychiatry 2001;158(11):1827-34.

21. Niethammer R, Weisbrod M, Schiesser S, Grothe J, Maier S, Peter U. Genetic Influence on Laterality in Schizophrenia? A Twin Study of Neurological Soft Signs. Am J Psychiatry 2000;157(2):272-4.

22. Marcus J, Hans SL, Byhouwer B, Norem J. Relationship among neurological functioning, intelligence quotients and physical anomalies. Schizophr Bull 1985;11(1):101-6.

23. Heuser M, Thomann PA, Essig M, Bachmann S, Schröder J. Neurological signs and morphological cerebral changes in schizophrenia: An analysis of NSS subscales in patients with first episode psychosis. Psychiatry Res 2011;192(2):69-76.

24. Dazzan P, Morgan KD, Orr KG, Hutchinson G, Chitnis X, Suckling J, Fearon P. The structural brain correlates of neurological soft signs in AESOP first-episode psychoses study. Brain 2004;127:143-53.

25. Lloyd T, Dazzan P, Dean K, Park SB, Fearon P, Doody GA. Minor physical anomalies in patients with firstepisode psychosis: their frequency and diagnostic specificity. Psychol Med 2008;38(1):71-7.

26. Fatemi SH, FolsomDT.The Neurodevelopmental Hypothesis of Schizophrenia, Revisited. Schizophr Bull 2009; 35(3):528-48.

27. Neelam K, Garg D, Marshall M. A systematic review and meta-analysis of neurological soft signs in relatives of people with schizophrenia. BMC Psychiatry 2011;11:139.

\author{
$* * * * * * * * * * * * * * * * * * * * * * * * * * * * * * * * * * *$ \\ Acknowledgements - Nil; \\ Conflict of Interest - Nil; \\ Funding - Nil.
}

